Published in final edited form as:

J Periodontol. 2005 November ; 76(11 Suppl): 2168-2174.

\title{
Resolution of Inflammation in Periodontitis
}

\author{
Alpdogan Kantarci ${ }^{*}$ and Thomas E. Van Dyke* \\ * Department of Periodontology and Oral Biology, Boston University Goldman School of Dental \\ Medicine, Boston, MA.
}

\begin{abstract}
Chronic inflammatory illnesses such as diabetes, arthritis, and heart disease are now seen as problems that might have impacts on the periodontium, and reciprocal effects of periodontal diseases are being considered as factors potentially affecting the progression of these diseases. Successful management of the inflammatory disorders in the human body depends on the identification of common pathways that would lead to a better understanding of the disease processes and development of novel treatment strategies. In this review, our objective is to identify the inflammatory basis of periodontal disease and common inflammatory mechanisms underlying several disorders elsewhere in the body, with an emphasis on how the potential extrinsic and intrinsic control methods could be used to prevent or treat the harmful effects linked to inflammation.
\end{abstract}

\section{Keywords}

Inflammation; lipoxin; periodontitis

\begin{abstract}
Historically, the separation of medicine and dentistry dates back to 1840, when the world's first dental school was established in Baltimore. ${ }^{1}$ Up to that point, dentistry and the treatment of oral disease were a discipline represented by poorly trained barber surgeons. ${ }^{2}$ Dentistry's emergence as a separate discipline led the profession to become an art and science, similar to medicine. Since the first medical texts (dating back to Huang Dee in Nai-Ching, China in 2,600 BC), it has been asserted that a "doctor" should attempt to "prevent" the "disease" before it requires "treatment". This approach dominated many early medical practices, but with the development of modern medicine, somewhat lost its importance in medical teachings. Until the reemergence of preventive and evidence-based medicine, medical professionals aimed to "treat" the disease and its outcomes. This philosophy of treatment was clearly based on faulty logic, however, since disease treatment incurs much higher expenses than cheaper and easier preventive measures. Thus, modern dental medicine, like other areas of modern medical science, has adopted "prevention of disease" as the main thrust. Yet, despite the growing congruence between dentistry and other medical science, drawbacks stemming from their historical separation are still evident. One of the primary remaining drawbacks is a continued apathy of the impact of systemic disease on the oral cavity and, more importantly, the impact of oral disease on systemic health. With the exception of Hunter's theory of focal infection in the early $20^{\text {th }}$ century, the oral cavity has essentially evolved in perception as an isolated organ system, without a significant impact on systemic health. ${ }^{2}$ Only in last decade has research emphasis on the significance of oral disease related to systemic health emerged. ${ }^{3}$ Chronic inflammatory illnesses such as diabetes, arthritis, and heart disease are now seen as problems that might have an impact on the periodontium, and reciprocal effects of periodontal diseases
\end{abstract}

Correspondence to: Thomas E. Van Dyke.

Correspondence: Dr. Thomas E. Van Dyke, Boston University Goldman School of Dental Medicine, Department of Periodontology and Oral Biology, 100 E. Newton St., G-05, Boston, MA 02118. Fax: 617/638-4799; e-mail: tvandyke@ bu.edu.. 
are being considered as factors potentially affecting the progression of these diseases. Thus, the next steps toward successful management of the inflammatory disorders in the human body depend on identification of common pathways that would lead to a better understanding of the disease processes and development of novel treatment strategies. ${ }^{4}$ Here, we aim to identify the inflammatory basis of periodontal disease and common inflammatory mechanisms underlying several disorders elsewhere in the body, with an emphasis on how potential extrinsic and intrinsic control methods could be used to prevent or treat the harmful effects linked to inflammation.

\section{“PUS BONUM ET LAUDABLE” (GOOD AND LAUDABLE PUS) OR THE "SECRET KILLER"?}

Which of these labels better describes inflammation? How much inflammation is beneficial? When does the inflammation start becoming a destructive process rather than a defense mechanism? Many diseases are being linked to excessive or uncontrolled inflammatory responses as the result of an ever-increasing interest in this field of study. Even in ancient times, the importance of pus as a "beneficial" substance was noted; hence, the ancient Latin name "good and laudable pus," which referred to the whitish creamy pus (therefore rich in polymorphonuclear leukocytes) that was a "good or preferable" response, as opposed to a thin or malodorous pus that suggested poor defense and/or highly vicious bacteria. ${ }^{5-7}$ The cover of the February 23, 2004, issue of Time magazine portrayed inflammation as playing a central role in many prevalent diseases (a "secret killer") such as Alzheimer's disease, cardiovascular disease, cancer, arthritis, and periodontal disease. ${ }^{4,12}$ This could be regarded as a paradigm shift in understanding many diseases that previously were categorized as infectious, metabolic, genetic, age-related, or linked to unknown etiologies.

Inflammation is defined as "a complex reaction to injurious agents such as microbes and damaged, usually necrotic, cells that consists of vascular responses, migration and activation of leukocytes, and systemic reactions." ${ }^{13}$ Thus, by definition, inflammation involves simultaneous processes of destruction and repair. While a fundamentally protective response, inflammation is also potentially deleterious. Recognition of self-destructive effects of inflammatory processes led to strategies of controlling inflammation through pharmacological and medical mechanisms. These methods, ideally, should present minimum unwanted effects that will not interfere with the protective properties of inflammatory cascades.

Inflammatory responses typically involve a series of reactions of blood vessels, leading to the accumulation of fluid and leukocytes in extravascular tissues. ${ }^{13}$ Inflammation serves to destroy, dilute, or eliminate the injurious agent while initiating events for healing and reconstruction of the damaged tissues. Biologically, inflammatory responses consist of vascular and cellular reactions through which many tissues and cells, fluid, and plasma proteins play interactive roles at various stages. Neutrophils, monocytes, eosinophils, lymphocytes, basophils, and platelets are circulating cells; mast cells surround blood vessels, and fibroblasts and macrophages are the resident connective tissue cells. Metchnikoff presented the importance of the phagocyte in host defense and inflammation more than 100 years ago. ${ }^{14,15}$ Since then, the focus of research in inflammation has evolved to elucidate the chemical mediators that could cause or mediate the cardinal signs of inflammation - heat, redness, swelling, pain, and loss of function in order to design "inhibitors of inflammation" and develop new treatments to control the unwanted side effects of inflammation. ${ }^{10,16}$ Numerous potential chemical mediators, such as lipids, proteins, cytokines, chemokines, growth factors, enzymes, and reactive oxygen species, as well as the many other radicals derived from gases, such as peroxynitrate, have been identified as useful for this purpose. ${ }^{17}$ Inflammation starts with an acute reaction, which evolves into a chronic phase if allowed to persist unresolved. Acute inflammation is a rapid onset, relatively short process characterized by fluid exudation and 
emigration of leukocytes, primarily neutrophils, whereas chronic inflammation extends over a longer time period and is associated with lymphocyte and macrophage infiltration, blood vessel proliferation, and fibrosis. Inflammation is terminated when the invader is eliminated and the secreted mediators are removed; however, many factors modify the course and morphologic appearance as well as the termination pattern and duration of inflammation.

\section{LIPID MEDIATORS OF INFLAMMATION}

Lipid mediators of platelet activating factor (PAF), lysolipids, and many known eicosanoids from arachidonic acid, including prostanoids, leukotrienes, and related compounds, play important roles in initiation and progression of inflammation and are thus termed "proinflammatory mediators." "10 18 Interactions between cells and at the transcellular level amplify and generate lipid-derived mediators, particularly those produced by lipoxygenases (Fig. 1). In addition to the proinflammatory mediators, "anti-inflammatory mediators" are also produced during these interactions. For example, lipoxins (LX) are formed from endogenous sources of arachidonate across many species, from fish to humans, in vivo. ${ }^{4},{ }^{10}{ }_{12} 26$ Thus, the major routes and biological actions of LX, aspirin-triggered 15-epi-lipoxins (ATLs), resolvins, docosatrienes, and neuroprotectins, can have therapeutic potential in regulating inflammation and diseases.

The local response to acute inflammation is an active rather than a passive process that triggers specific biochemical and cellular programs of resolution. ${ }^{27}$ The first "pro-resolution" pathway involves novel lipid mediators that possess endogenous anti-inflammatory and pro-resolution properties that are termed as resolvins. ${ }^{10,2}$ In addition, novel lipid mediators produced from docosahexaenoic acid (DHA), an abundant omega-3 fatty acid in neural tissues, are now coined as docosatrienes, and $17 \mathrm{~S}$ series resolvins were found to be both anti-inflammatory and tissue protective. ${ }^{29.33}$ It is clear that future care of trauma and surgical patients, as well those with periodontal diseases, will rely heavily on clinicians having a detailed map and fundamental appreciation of the temporal relationships involved in the resolution program of local acute inflammation and tissue injury at the cellular, molecular, organ, and systems levels.

Although intended for host defense, professional phagocytes can amplify injury via release of proinflammatory mediators. This could explain the pathophysiology observed in many clinical as well as chronic inflammatory diseases, such as arthritis. Acute inflammation and its timely resolution play central roles in the body's response to trauma, tissue injury, ischemiareperfusion, and surgical interventions, as well as in microbial host defense. ${ }^{4}$ Lipid-derived mediators such as the eicosanoids, particularly classic prostaglandins (PG) and leukotrienes (LT), play pivotal roles in orchestrating inflammation. These are autacoids or local-acting mediators within the innate acute inflammatory response. ${ }^{34}$ Nonsteroidal anti-inflammatory drugs (NSAID) such as aspirin and cyclooxygenase inhibitors (both COX-1 and -2) are used to block PG biosynthesis. ${ }^{34} 36$ These drugs are already in use and appear to be effective in many clinical settings, although there is considerable controversy in the clinical literature about the general safety and unwanted side effects of $\mathrm{COX}_{37}-2$ inhibitors in the gastrointestinal and cardiovascular systems as well as about their cost.

Any current anti-inflammatory medicines used clinically today are not free of unwanted side effects. The approach for making one "super-inhibitor" or antagonist to block inflammation yet leave resolution intact appears to be difficult or perhaps even flawed for two primary reasons. First, there is an enormous redundancy in the number of "proinflammatory" mediators and apparently no obvious master switch or magic bullet. Second, current anti-inflammatory agents, COX-1 and -2 inhibitors, glucocorticoids, and PG themselves and their degradative enzymes can dysregulate resolution and prolong inflammation. Finally, there seems to be a novel link between host defense anti-inflammatory lipid mediators, namely that LX and ATL 
activate antimicrobial defense mechanisms in epithelial cells. ${ }^{40}$ Thus, there remain considerable opportunities for improvement in the management of trauma-related injury and acute inflammation as well as chronic inflammatory diseases such as periodontal diseases via novel anti-inflammatory pro-resolving therapies. Novel lipid mediator pathways are very attractive for new therapeutic interventions because they are: 1) small molecules ( $<500 \mathrm{MW}$ ); 2) amenable to total organic synthesis; and 3) manufacturable with currently available pharmaceutical facilities. ${ }^{30}, 41$

\section{ROLE OF PHAGOCYTES IN INFLAMMATION}

While it is well appreciated today that neutrophils play an essential role in host defense, their aberrant and prolonged activation can lead to the tissue injury and deleterious sequela observed in many common chronic disease phenotypes. Trauma, injury, and surgical treatments can also lead to injury from within, where activated neutrophils are central in exacerbating the injury by releasing noxious agents. Recently, we have shown that localized aggressive periodontitis is an example of neutrophil-mediated tissue destruction. ${ }^{42,43}$ Local tissue inflammation is a physiologic event in which there are both positive and negative signals. Because of the importance of leukocyte trafficking, these can be referred as local "go" and "stop" signals, ${ }^{13,44}$ endogenous mediators of anti-inflammation and pro-resolution, ${ }^{45} 48$ or "checkpoint regulators."

A constant feature of acute inflammation is that polymorphonuclear leukocytes (PMN) arrive at the scene first and mononuclear cells arrive next. ${ }^{5,13}$ In experimental models of inflammation, where it was well documented that PMN precede the recruitment of mononuclear cells and then disappear as the mono-nuclear cell prepares for self-limited resolution and retraction to granulation tissue, specialized circuits within resolution exist (Fig. 2). Until recently, however, few studies focused on the impact of common and widely used drugs within this sequential resolution. It has been now shown that there is a temporal dissociation between eicosanoids, where PG and LT antecede the biosynthetic assembly of the enzymes required for the production of $\mathrm{LX}_{49}$ and these studies open up the possibility that other pathways are functional within resolution. ${ }^{49}$ The potential beneficial impact of essential omega-3 fatty acids was suspected as early as 1929 from the studies of Burr and Burr. ${ }^{50}$ Novel oxygenated products generated from precursors EPA and DHA that possessed potent bioactions were identified in resolving inflammatory exudates, and similar structures were elucidated in tissues rich in DHA. Hence, the terms "resolvins" (resolution phase interaction products) and "docosatrienes" were introduced in initial studies because they displayed potent anti-inflammatory and immunoregulatory properties. Unlike other products identified earlier from omega-3 fatty acids that are similar in structure to eicosanoids but less potent or devoid of bioactions, the resolvins, docosatrienes, and neuroprotectins evoke potent biological actions in vitro and in vivo. Specific receptors for these novel omega-3 bioactive products are abbreviated Reso (E or D) receptors to designate the precursor origin and a numerical index (i.e., ResoE1) in recognition of their respective cognate ligands. These novel bio-active epimers are denoted as aspirin-triggered (AT)-RvDs and AT-DTs (Fig. 3).

Another important and well-characterized anti-inflammatory lipid mediator is $\mathrm{LXA}_{4}$. $\mathrm{LXA}_{4}$ is produced in vivo during the course of inflammation as in experimental immune complex glomerulonephritis model and in pleural exudate upon allergen challenge in rats. Endogenous $\mathrm{LXA}_{4}$ is also produced in ischemic lungs and elevated by reperfusion in a hind limb ischemia reperfusion model. In addition, $\mathrm{LXA}_{4}$ is formed in rat brain and elevated in focal cerebral ischemia (reviewed in references 10 and ${ }^{25}$ ). In localized aggressive periodontitis, $\mathrm{LXA}_{4}$ production is upregulated. ${ }^{51}$ This is the same case with mild asthma, as well as following atherosclerotic plaque rupture and with nasal polyps. Moreover, ATL and $\mathrm{LXA}_{4}$ formation was evaluated in aspirin-tolerant and aspirin-intolerant asthmatics. Together, these results 
indicate that alterations in LX and ATL levels may be linked to the pathophysiology of several human diseases and may display local organ-specific functions that stand apart from their roles in inflammation and within local inflammatory lesions. $\mathrm{LXA}_{4}$ and ATL display counterregulatory roles in various cell types in vitro. With human peripheral blood leukocytes, $\mathrm{LXA}_{4}$ inhibits both isolated PMN and eosinophil chemotaxis in vitro in the nanomolar range and blocks human natural killer cell cytotoxicity in a stereoselective fashion, as well as stimulating myeloid bone marrow-derived progenitors. $\mathrm{LXA}_{4}$ stimulates chemotaxis and adherence in monocytes, but there are no apparent "proinflammatory" responses of these cells in vitro or in vivo. This finding that may relate to the recruitment of monocytes to sites of wound healing and clearance. Indeed, LX and ATL stimulate the uptake of apoptotic PMN by macrophages.

In dermal inflammation, LX stable analogs, when applied topically to mouse ears, inhibit both PMN infiltration and vascular permeability changes. ${ }^{32}$ Inhibition was evidenced by either local intra-air pouch delivery or via systemic delivery by intravenous injection and proved more potent than local delivery of aspirin. Recently, using a casein-induced peritonitis model in rats, ATL was shown to significantly inhibit neutrophil infiltration $(\sim 43 \%)$ and protein extravasation $(\sim 42 \%)$ when given intravenously with two consecutive doses at approximately $60 \mathrm{mg} / \mathrm{kg}$ per injection. ${ }^{53}$ Transgenic expression of the human ALX (namely, the human receptor expressed in the mouse model) gives markedly decreased PMN infiltration to the peritoneum in zymosan-initiated peritonitis and displayed increased sensitivity to suboptimal doses of ATL stable analog. ${ }^{54}$ ATL analogs were also shown to rapidly promote macrophage phagocytosis of apoptotic PMN in a thioglycollate-induced peritonitis, supporting a role for LX as pro-resolution signals in inflammation.

\section{RESOLUTION OF PERIODONTAL INFLAMMATION AND ITS EFFECT ON SYSTEMIC DISEASE RESOLUTION}

Recently, we have demonstrated that transgenic rabbits overexpressing 15-LO generate enhanced levels of LX, have an enhanced anti-inflammatory status, and are protected from the inflammatory bone loss of periodontal disease. ${ }^{56}$ In the first of these series of studies, leukocytes from 15-LO TG rabbits exhibited enhanced LX production, underscoring differences in lipid mediator profiles compared with non-TG rabbits. P. gingivalis-associated inflammation and leukocyte-mediated bone destruction were assessed by initiating acute periodontitis. 15-LO TG rabbits exhibited markedly reduced bone loss and local inflammation (Fig. 4). Because enhanced LX production was associated with an increased anti-inflammatory status of 15-LO TG rabbits, a stable analog of $\mathrm{LXA}_{4}$ was applied to the gingival crevice subject to periodontitis. Topical application of 15-epi-16-phenoxy-para-fluoro- $\mathrm{LXA}_{4}$ stable analog (ATLa) dramatically reduced leukocyte infiltration, ensuing bone loss, as well as inflammation. These results indicate that overexpression of $15-\mathrm{LO}$ and $\mathrm{LXA}_{4}$ is associated with dampened PMN-mediated tissue degradation and bone loss, suggesting that enhanced anti-inflammation status is an active process. ${ }^{56}$ Moreover, the findings also suggested that LX have potential for novel approaches to diseases, e.g., periodontitis and arthritis, where inflammation and bone destruction are features.

\section{CONCLUSIONS}

Lipoxins, resolvins, and docosatrienes are novel families comprised of separate chemical series of lipid-derived mediators, each with unique structures and apparent complementary antiinflammatory properties and actions. These families of compounds can also be generated in their respective epimeric forms when aspirin is given in mammalian systems. The resolvins and docosatrienes dampen from within both inflammation and PMN-mediated injury, key culprits in many human diseases. It is likely that these compounds and their AT-related forms 
may play roles in other tissues and organs, since they are involved in physiological and pathological processes. These novel lipid mediators are evolutionally conserved molecules that are host protective. In view of the important roles of their precursors DHA and EPA in human biology and medicine, it is likely that these novel pathways and compounds are responsible in part for the beneficial impact of omega-3 essential fatty acids in complex systems and could be of use in periodontal treatment.

\section{REFERENCES}

1. Ring, ME. Dentistry: An Illustrated History. Abrams; New York: 1985. p. 212

2. Rutkauskas JS. The medical necessity of periodontal care. Periodontol 2000 2000;23:151-156. [PubMed: 11276763]

3. Kinane DF, Lowe GDO. How periodontal disease may contribute to cardiovascular disease. Periodontol 2000 2000;23:121-126. [PubMed: 11276758]

4. Van Dyke TE, Serhan CN. Resolution of inflammation: A new paradigm for the pathogenesis of periodontal diseases. J Dent Res 2003;82:82-90. [PubMed: 12562878]

5. Majno G. Inflammation and infection: Historic highlights. Monogr Pathol 1982;23:1-17. [PubMed: 6750363]

6. Ryan GB, Majno G. Acute inflammation. A review. Am J Pathol 1977;86:183-276. [PubMed: 64118]

7. Majno G. Chronic inflammation: Links with angiogenesis and wound healing. Am J Pathol 1998;153:1035-1039. [PubMed: 9777935]

8. Gorman C, Park A, Dell K. The Fires Within. Time 2004;163:8.

9. Helgadottir A, Manolescu A, Thorleifsson G, et al. The gene encoding 5-lipoxygenase activating protein confers risk of myocardial infarction and stroke. Nat Genet 2004;36:233-239. [PubMed: 14770184]

10. Serhan CN. A search for endogenous mechanisms of anti-inflammation uncovers novel chemical mediators: Missing links to resolution. Histochem Cell Biol 2004;122:305-321. [PubMed: 15322859]

11. Pasche B, Serhan CN. Is C-reactive protein an inflammation opsonin that signals colon cancer risk? JAMA 2004;291:623-624. [PubMed: 14762043]

12. Erlinger TP, Platz EA, Rifai N, Helzlsouer KJ. C-reactive protein and the risk of incident colorectal cancer. JAMA 2004;291:585-590. [PubMed: 14762037]

13. Kumar, V.; Abbas, AK.; Fausto, N. Robbins and Cotran Pathologic Basis of Disease. 7th. Elsevier Saunders; Philadelphia: 2005. p. 48-85.

14. Tauber AI, Chernyak L. The birth of immunology. II. Metchnikoff and his critics. Cell Immunol 1989;121:447-473. [PubMed: 2661021]

15. Chernyak L, Tauber AI. The birth of immunology: Metchnikoff, the embryologist. Cell Immunol 1988;117:218-233. [PubMed: 3052859]

16. Winyard G. Doctors, managers and politicians. Clin Med 2003;3:465-469. [PubMed: 14601948]

17. Nathan C. Points of control in inflammation. Nature 2002;420:846-852. [PubMed: 12490957]

18. Samuelsson B. The leukotrienes: An introduction. Adv Prostaglandin Thromboxane Leukot Res 1982;9:1-17. [PubMed: 6124094]

19. Fiorucci S, Wallace JL, Mencarelli A, et al. A beta-oxidation-resistant lipoxin A4analog treats hapteninduced colitis by attenuating inflammation and immune dysfunction. Proc Natl Acad Sci USA 2004;101:15736-15741. [PubMed: 15505205]

20. Fiorucci S, Distrutti E, Mencarelli A, et al. Cooperation between aspirin-triggered lipoxin and nitric oxide (NO) mediates antiadhesive properties of 2-(Acetyloxy)-benzoic acid 3-(nitrooxymethyl) phenyl ester (NCX-4016) (NO-aspirin) on neutrophil-endothelial cell adherence. J Pharmacol Exp Ther 2004;309:1174-1182. [PubMed: 14762100]

21. Fiorucci S, Santucci L, Wallace JL, et al. Interaction of a selective cyclooxygenase-2 inhibitor with aspirin and NO-releasing aspirin in the human gastric mucosa. Proc Natl Acad Sci USA 2003;100:10937-10941. [PubMed: 12960371] 
22. Fiorucci S, Distrutti E, Mencarelli A, et al. Evidence that 5-lipoxygenase and acetylated cyclooxygenase 2-derived eicosanoids regulate leukocyte-endothelial adherence in response to aspirin. Br J Pharmacol 2003;139:1351-1359. [PubMed: 12890715]

23. Fiorucci S, de Lima OM Jr, Mencarelli A, et al. Cyclooxygenase-2-derived lipoxin $\mathrm{A}_{4}$ increases gastric resistance to aspirin-induced damage. Gastroenterology 2002;123:1598-1606. [PubMed: 12404234]

24. Wallace JL, Vergnolle N, Muscara MN, et al. Enhanced anti-inflammatory effects of a nitric oxidereleasing derivative of mesalamine in rats. Gastroenterology 1999;117:557-566. [PubMed: 10464131]

25. Kantarci A, Van Dyke TE. Lipoxins in chronic inflammation. Crit Rev Oral Biol Med 2003;14:412. [PubMed: 12764016]

26. Aliberti J, Serhan C, Sher A. Parasite-induced lipoxin $\mathrm{A}_{4}$ is an endogenous regulator of IL-12 production and immunopathology in Toxoplasma gondii infection. J Exp Med 2002;196:1253-1262. [PubMed: 12417634]

27. Serhan CN, Oliw E. Unorthodox routes to prostanoid formation: New twists in cyclooxygenaseinitiated pathways. J Clin Invest 2001;107:1481-1489. [PubMed: 11413151]

28. Arita M, Bianchini F, Aliberti J, et al. Stereochemical assignment, antiinflammatory properties, and receptor for the omega-3 lipid mediator resolvin E1. J Exp Med 2005;201:713-722. [PubMed: 15753205]

29. Hong S, Gronert K, Devchand PR, Moussignac RL, Serhan CN. Novel docosatrienes and 17Sresolvins generated from docosahexaenoic acid in murine brain, human blood, and glial cells. Autacoids in anti-inflammation. J Biol Chem 2003;278:14677-14687. [PubMed: 12590139]

30. Serhan CN, Hong S, Gronert K, et al. Resolvins: A family of bioactive products of omega-3 fatty acid transformation circuits initiated by aspirin treatment that counter proinflammation signals. J Exp Med 2002;196:1025-1037. [PubMed: 12391014]

31. Marcheselli VL, Hong S, Lukiw WJ, et al. Novel docosanoids inhibit brain ischemia-reperfusionmediated leukocyte infiltration and pro-inflammatory gene expression. J Biol Chem 2003;278:43807-43817. [PubMed: 12923200]

32. Serhan CN, Arita M, Hong S, Gotlinger K. Resolvins, docosatrienes, and neuroprotectins, novel omega-3-derived mediators, and their endogenous aspirin-triggered epimers. Lipids 2004;39:11251132. [PubMed: 15726828]

33. Bannenberg GL, Chiang N, Ariel A, et al. Molecular circuits of resolution: Formation and actions of resolvins and protectins. J Immunol 2005;174:4345-4355. [PubMed: 15778399]

34. Samuelsson B, Dahlén S-E, Lindgren JÅ, Rouzer CA, Serhan CN. Leukotrienes and lipoxins: Structures, biosynthesis, and biological effects. Science 1987;237:1171-1176. [PubMed: 2820055]

35. Flower RJ. Studies on the mechanism of action of anti-inflammatory drugs: A paper in honour of John Vane. Thromb Res 2003;110:259-263. [PubMed: 14592544]

36. Drazen JM, Israel E, O'Byrne PM. Treatment of asthma with drugs modifying the leukotriene pathway. N Engl J Med 1999;340:197-206. [PubMed: 9895400]

37. Jackson LM, Hawkey CJ. COX-2 selective nonsteroidal anti-inflammatory drugs. Do they really offer any advantages? Drugs 2000;59:1207-1216. [PubMed: 10882157]

38. Mamdani M, Rochon P, Juurlink DN, et al. Effect of selective cyclooxygenase 2 inhibitors and naproxen on short-term risk of acute myocardial infarction in the elderly. Arch Intern Med 2003;163:481-486. [PubMed: 12588209]

39. Mengle-Gaw LJ, Schwartz BD. Cyclooxygenase-2 inhibitors: Promise or peril? Mediators Inflamm 2002;11:275-286. [PubMed: 12467519]

40. Levy O, Canny G, Serhan CN, Colgan SP. Expression of BPI (bactericidal/permeability-increasing protein) in human mucosal epithelia. Biochem Soc Trans 2003;31:795-800. [PubMed: 12887308]

41. Navia MA, Peattie DA. Structure-based drug design: Applications in immunopharmacology and immunosuppression. Immunol Today 1993;14:296-302. [PubMed: 8397770]

42. Gronert K, Kantarci A, Levy BD, et al. A molecular defect in intracellular lipid signaling in human neutrophils in localized aggressive periodontal tissue damage. J Immunol 2004;172:1856-1861. [PubMed: 14734770] 
43. Kantarci A, Oyaizu K, Van Dyke TE. Neutrophil-mediated tissue injury in periodontal disease pathogenesis: Findings from localized aggressive periodontitis. J Periodontol 2003;74:66-75. [PubMed: 12593599]

44. Brady HR, Serhan CN. Lipoxins: Putative braking signals in host defense, inflammation and hypersensitivity. Curr Opin Nephrol Hypertens 1996;5:20-27. [PubMed: 8834158]

45. Serhan CN. Eicosanoids in leukocyte function. Curr Opin Hematol 1994;1:69-77. [PubMed: 9371262]

46. Serhan CN, Fiore S, Levy BD. Cell-cell interactions in lipoxin generation and characterization of lipoxin $A_{4}$ receptors. Ann NY Acad Sci 1994;744:166-180. [PubMed: 7825838]

47. Serhan CN. Lipoxin biosynthesis and its impact in inflammatory and vascular events. Biochim Biophys Acta 1994;1212:1-25. [PubMed: 8155718]

48. Serhan CN, Fiore S. Lipoxin recognition sites of human neutrophils. Adv Prostaglandin Thromboxane Leukot Res 1994;22:317-326. [PubMed: 7771345]

49. Levy BD, Clish CB, Schmidt B, Gronert K, Serhan CN. Lipid mediator class switching during acute inflammation: Signals in resolution. Nat Immunol 2001;2:612-619. [PubMed: 11429545]

50. Burr GO, Burr MM. A new deficiency disease produced by the rigid exclusion of fat from the diet. J Biol Chem 1929;82:345.

51. Pouliot M, Clish CB, Petasis NA, Van Dyke TE, Serhan CN. Lipoxin A4 analogues inhibit leukocyte recruitment to Porphyromonas gingivalis: A role for cyclooxygenase- 2 and lipoxins in periodontal disease. Biochemistry 2000;39:4761-4768. [PubMed: 10769133]

52. Clish CB, O'Brien JA, Gronert K, Stahl GL, Petasis NA, Serhan CN. Local and systemic delivery of a stable aspirin-triggered lipoxin prevents neutrophil recruitment in vivo. Proc Natl Acad Sci USA 1999;96:8247-8252. [PubMed: 10393980]

53. Chiang N, Takano T, Arita M, Watanabe S, Serhan CN. A novel rat lipoxin $\mathrm{A}_{4}$ receptor that is conserved in structure and function. Br J Pharmacol 2003;139:89-98. [PubMed: 12746227]

54. Devchand PR, Arita M, Hong S, et al. Human ALX receptor regulates neutrophil recruitment in transgenic mice: Roles in inflammation and host defense. FASEB J 2003;17:652-659. [PubMed: 12665478]

55. Mitchell S, Thomas G, Harvey K, et al. Lipoxins, aspirin-triggered epi-lipoxins, lipoxin stable analogues, and the resolution of inflammation: Stimulation of macrophage phagocytosis of apoptotic neutrophils in vivo. J Am Soc Nephrol 2002;13:2497-2507. [PubMed: 12239238]

56. Serhan CN, Jain A, Marleau S, et al. Reduced inflammation and tissue damage in transgenic rabbits overexpressing 15-lipoxygenase and endogenous anti-inflammatory lipid mediators. J Immunol 2003;171:6856-6865. [PubMed: 14662892] 


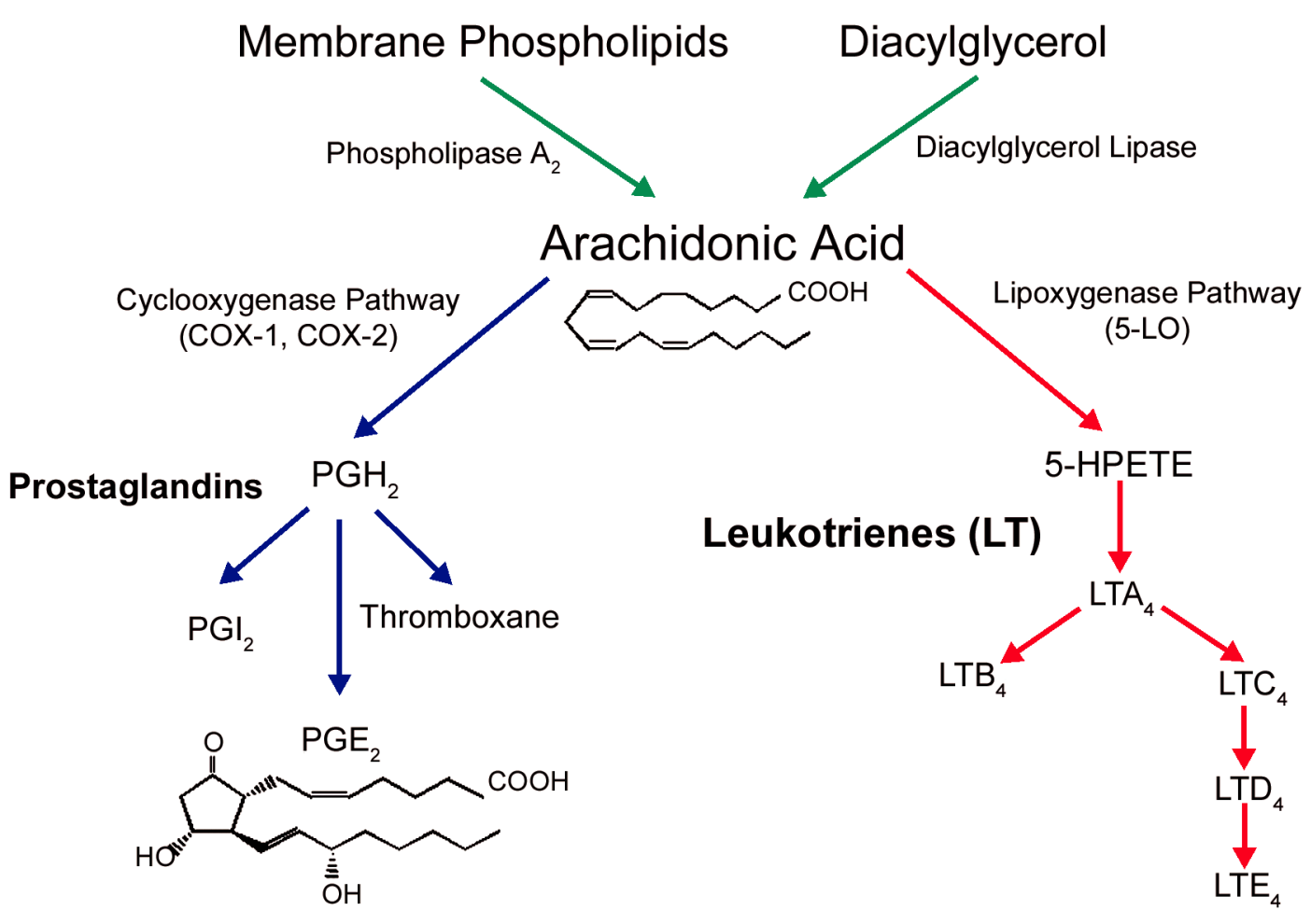

Figure 1.

Mediators of arachidonic acid metabolism lead to generation of prostaglandins (PG) and leukotrienes (LT) through cyclooxygenase (COX) and lipoxygenase (LO) pathways. 


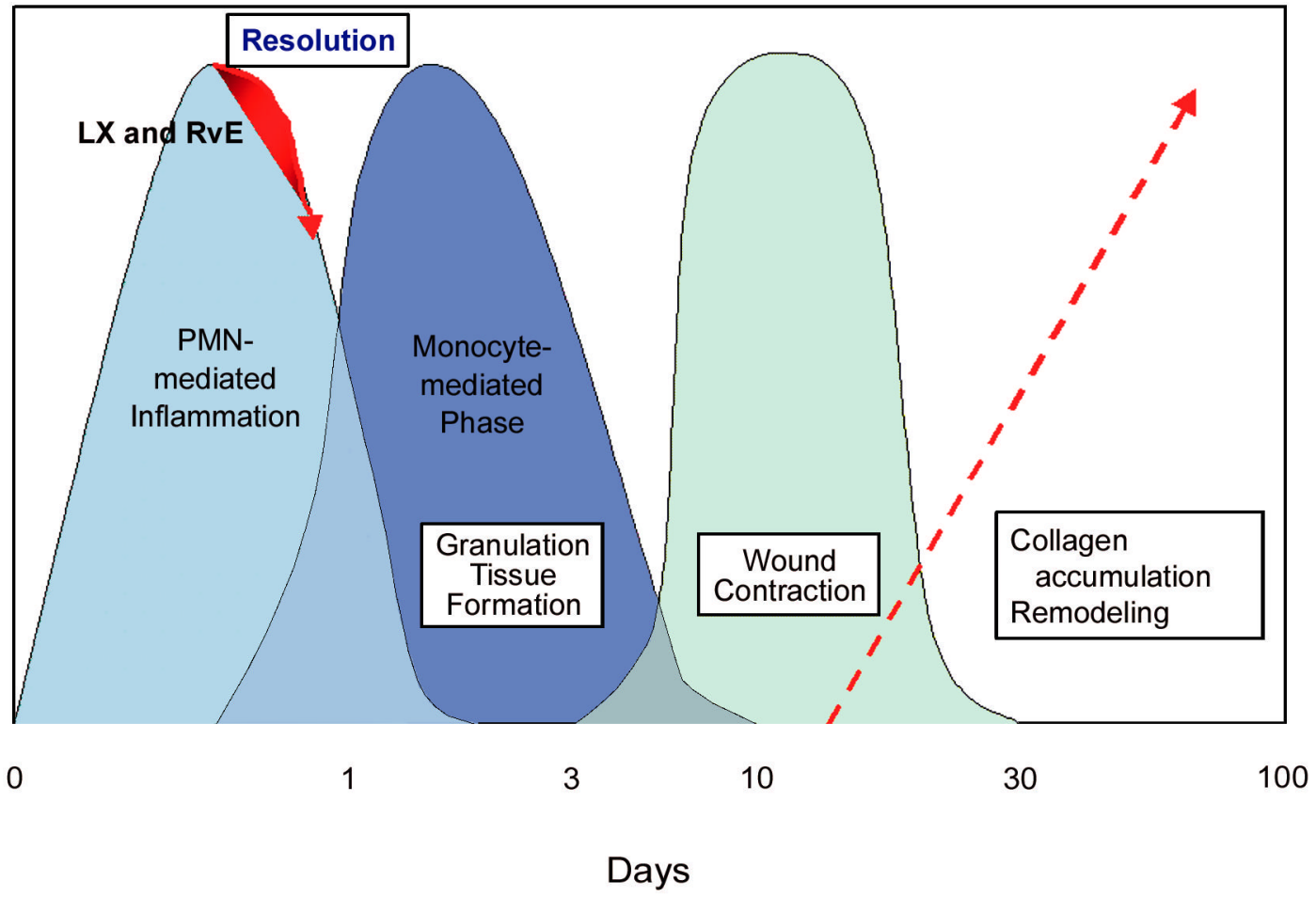

Figure 2.

Sequential events in inflammation. PMN-mediated inflammation is the first step in host response against infection. This phase is followed by monocyte-dominated events during which granulation tissue formation occurs. Stop signals of LX and RvE prevent excessive PMNmediated tissue response to stimuli. 

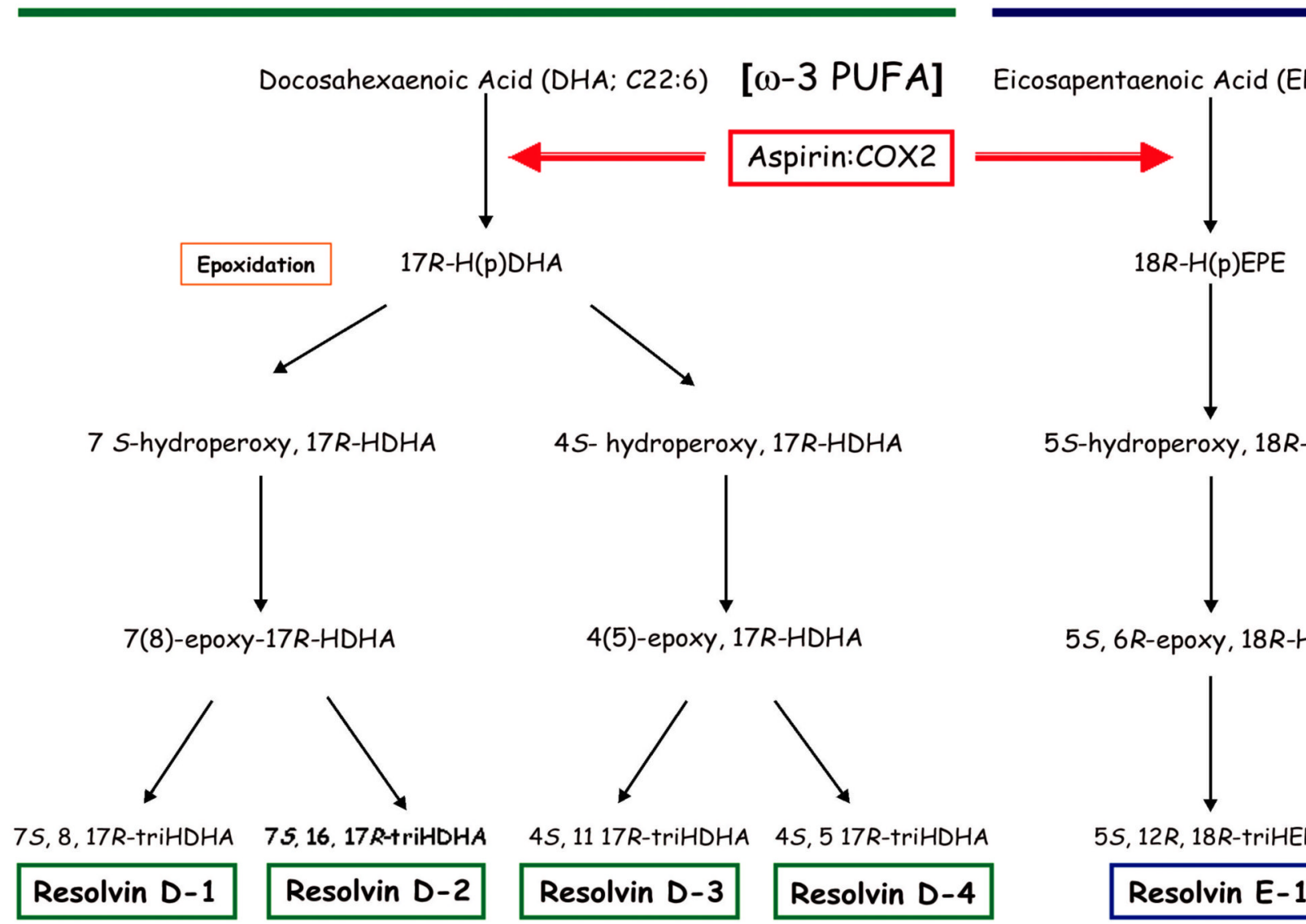

5S-hydroperoxy, 18R-HEPE

Figure 3.

Biosynthesis of resolvins. Omega- 3 polyunsaturated fatty acids ( $\omega-3$ PUFA) such as docosahexaenoic acid (DHA) and eicosapentaenoic acid (EPA) are metabolized by the acetyl salisilic acid (aspirin) acetylation of cyclooxygenase (COX)-2 into resolvins. Human PMN through the action of COX-2 converts DHA into 13-hydroxy-DHA, which is switched to 17RHDHA through aspirin. This molecule is transformed into two compounds via 5lipoxygenation that are each rapidly transformed into two intermediates. These two novel epoxide intermediates open to bioactive trihydroxy-containing products denoted 17R series Resolvin D-1, Resolvin D-2, Resolvin D-3, and Resolvin D-4. Meanwhile, endothelial cells that express COX-2 will convert EPA into 18R-H(p)EPE in the presence of aspirin, and this line of molecules will give rise to Resolvin-E1. 


\section{Ligature Alone}

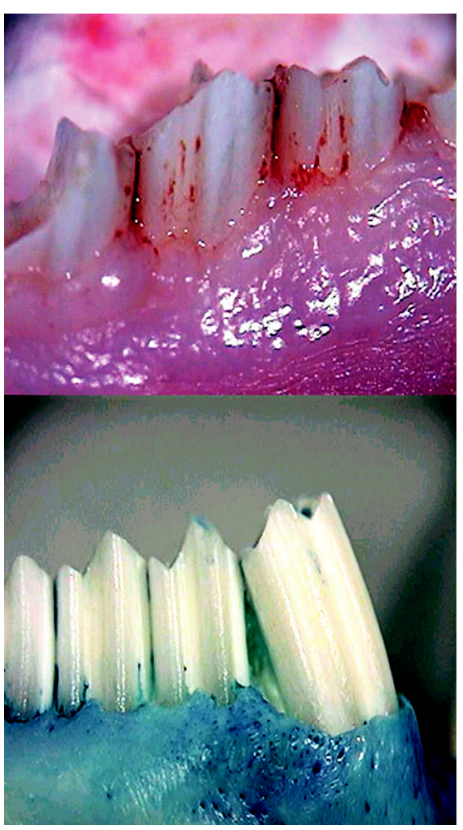

Ligature+ P.gingivalis

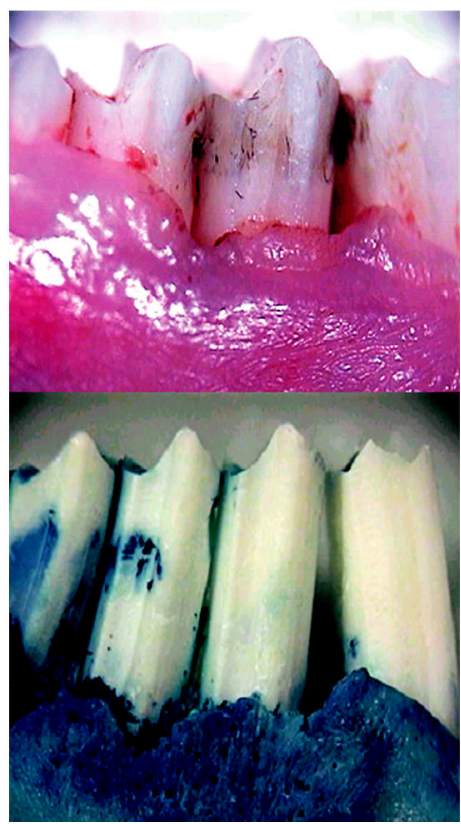

15-LO-Transgenic+ P.gingivalis

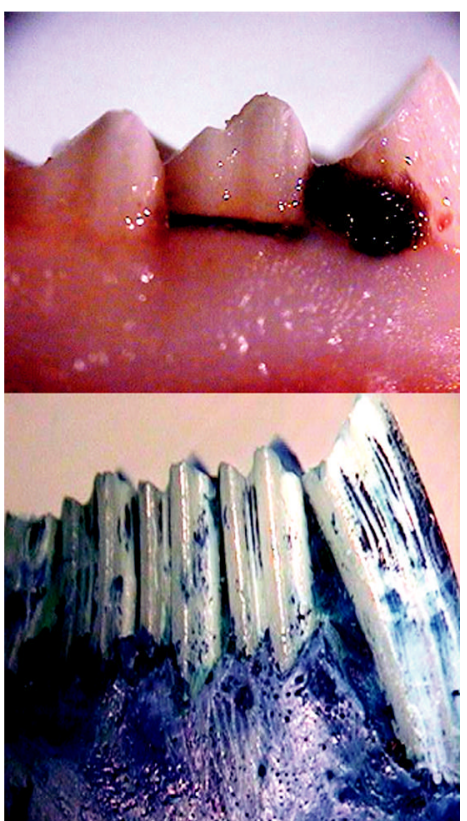

Figure 4.

15-LO transgenic rabbits exhibited no evidence of periodontitis. 